\title{
Novel Antiproliferative and Antioxidant Role of BjAnn1, a Mustard Annexin Protein in Human Glioblastoma Cell Lines
}

Gangadhara R Sareddy ${ }^{1 \#}$, Divya Kesanakurti", Pulugurtha Bharadwaja Kirti ${ }^{\text {** }}$ and Phanithi Prakash Babu ${ }^{1 *}$

${ }^{1}$ Department of Biotechnology, School of Life Sciences, University of Hyderabad, Hyderabad, India

${ }^{2}$ Department of Cancer Biology and Pharmacology, University of Illinois College of Medicine, Peoria, IL, USA

${ }^{3}$ Department of Plant Sciences, School of Life Sciences, University of Hyderabad, Hyderabad, India

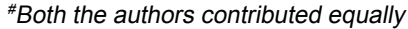

\begin{abstract}
Glioblastoma represents the deadliest tumors of the central nervous system and their bleak prognosis remains unchanged over the past few decades and requiring new treatments that curb the tumor progression. Cancer cells possess high reactive oxygen species (ROS) levels when compared to their normal control counterparts. We have earlier characterized the ROS-scavenging annexin protein, BjAnn1 from Brassica juncea and demonstrated its peroxidase activity. Since annexins are widely conserved across evolutionary lines with involvement in stress alleviation, we attempted to verify the effect of ectopic expression of ROS-scavenging BjAnn1 in human glioblastoma cell lines. The multiple sequence alignment revealed that BjAnn1 showed homology to human annexin A13 (73.18\%), annexin A2 (70.66\%), annexin A3 (72.53\%) and annexin A8 (70.03\%). Stable expression of BjAnn1 in U251 and U87 cells inhibited proliferation, migration and invading abilities in correlation with significant decrease in ROS levels in comparison to the empty vector (EV)-expressing controls. Multiple gene-profiling using cDNAPCR arrays revealed a prominent transcriptional upregulation of oxidative stress responsive genes including CCS, CYB, DUOX2, FOXM, GSS, MBL2, MT3, MTL5, NME5, PXDN, SOD2 and SOD3 in BjAnn1-expressing glioblastoma cells. Western blotting confirmed the significant increase in the expression of antioxidant enzymes SOD2 (MnSOD) and SOD3 (Cu/ZnSOD) in BjAnn1-expressing cells. We also observed a significant inhibition in nuclear translocation of p65 and p50, NF-KB reporter activity and expression of NF-kB-target genes in BjAnn1 expressing cells. In summary, our study shows a prominent tumor-suppressive role of ROS-scavenging BjAnn1 in glioblastoma cell lines suggesting it as a novel candidate for efficient gene therapy in glioblastoma.
\end{abstract}

Keywords: Glioblastoma; Plant annexin; Oxidative stress; Antioxidant enzymes; Reactive oxygen species

\section{Introduction}

Oxidative stress is caused due to the redox imbalance and excessive generation of reactive oxygen species (ROS) including superoxide $\left(\mathrm{O}^{2-}\right)$, hydrogen peroxide $\left(\mathrm{H}_{2} \mathrm{O}_{2}\right)$ and hydroxyl radical $\left(\mathrm{OH}^{-}\right)$as a result of various metabolic enzymatic processes in cells [1-3]. Although basal levels of ROS are required to regulate various signaling pathways in proliferating cells, excessive ROS accumulation induces deleterious modifications on essential macromolecules such as lipids, proteins and DNA leading to cellular damage [3]. To cope with high ROS, cells employ various defense mechanisms using antioxidant enzymes including catalases, peroxidases and superoxide dismutases [3-5]. However, several studies demonstrated the critical role of increased ROS levels in the tumor initiation and progression by increasing cell proliferation, migration and invasion [6]. The characteristic anchorage-independent growth and resistance towards anoikis-mediated cell death was ascribed to the high ROS levels and constant oxidative stress in tumor cells when compared to their normal counterparts [7,8]. High ROS levels were also implicated in the maintenance of stem-like characteristics of the cancer-initiating cells and anchorage-independent growth in the malignantly transformed invading cancer cells $[8,9]$.

Annexins are a superfamily of calcium-binding proteins that bind to negatively charged membrane phospholipids in cells and were reported to be conserved in eukaryotes [10-12]. Interestingly emerging evidences have shown that annexins are among the genes, whose expression is differentially altered in various neoplasms [13]. Annexin gene expression is differentially regulated by various stress stimuli and is reported to be involved in exocytosis, actin binding, ion transport, intracellular calcium signaling, growth regulation, apoptosis and inflammation, [14-17]. Several studies demonstrated the peroxidase activity of plant annexins and this activity was primarily attributed to the presence of sulfur cluster in the first annexin domain and conserved His 40 residue at the N-terminal region which is homologous to haembinding domain of horse-radish peroxidase, however, subsequent site-directed mutagenesis studies confirmed that His 40 is not essential for maintaining the annexin peroxidase activity [18-20]. Conversely, animal annexins lack His40 residue and were not reported to display any peroxidase activity until now. Upon heterologous expression, AnnAtl from Arabidopsis thaliana displayed peroxidase activity and rescued $\mathrm{H}_{2} \mathrm{O}_{2}$-sensitive E. coli $\Delta \mathrm{OxyR}$ mutant and mammalian cells from oxidative stress-induced cell death [21-23]. Our earlier studies demonstrated that the Brassica juncea annexin 1 protein (BjAnn1), the close homolog of AnnAt1, also exhibits in vitro peroxidase activity [24]. The transgenic overexpression of BjAnn1 in tobacco and cotton conferred tolerance towards multiple stresses including oxidative stress by scavenging stress-induced ROS formation [24-25].

*Corresponding authors: Phanithi Prakash Babu, Professor and Head Department of Biotechnology, School of Life Sciences, University of Hyderabad, Hyderabad, India-500046, Tel: 0091-40-23134584; 23013336; Fax: 0091-40 23010120; E-mail: prakashbabuphanithi@gmail.com, ppbsl@uohyd.ernet.in

Pulugurtha Bharadwaja Kirti, Department of Biotechnology, School of Life Sciences, University of Hyderabad, Hyderabad, India-500046, Tel: 0091-4023134584; 23013336; Fax: 0091-40-23010120

Received April 27, 2013; Accepted June 20, 2013; Published June 24, 2013

Citation: Gangadhara Reddy S, Divya K, Kirti PB, Prakash Babu P (2013) Nove Antiproliferative and Antioxidant Role of BjAnn1, a Mustard Annexin Protein in Human Glioblastoma Cell Lines. J Cancer Sci Ther 5: 256-263. doi:10.4172/1948 5956.1000214

Copyright: (c) 2013 Gangadhara Reddy S, et al. This is an open-access article distributed under the terms of the Creative Commons Attribution License, which permits unrestricted use, distribution, and reproduction in any medium, provided the original author and source are credited. 
Glioblastoma (GBM) are the most malignant, adult brain tumors and account for $\sim 80 \%$ of all primary brain tumors [26,27]. Despite modest improvement in the standard therapies, the median survival is approximately $12-15$ months after diagnosis [28]. Therefore, finding new therapeutic options against these aggressive tumors is clinically significant. Elevated ROS has been correlated with the activation of several signaling mechanisms essential for tumor initiation and progression including NF- $\mathrm{KB}$ pathway [29]. Constitutive activation of NF- $\kappa \mathrm{B}$ pathway is evident in glioblastomas and is associated with enhanced cell growth, cell cycle progression, chemoresistance, migration and invasion [30-32]. Previous reports showed the antiproliferative roles of antioxidant enzymes in cancer cell lines by detoxification of ROS [5,33,34]. Among these, manganese-superoxide dismutase (SOD2) was shown to be a tumor suppressor and implicated in oxidative stress response in various cancers $[7,35,36]$. Overexpression of SOD2 and SOD3 reduced the malignant phenotype and abrogated tumor growth in gliomas $[5,35]$.

Based on the significant sequence homology with human annexins, peroxidase and ROS-detoxifying activities of BjAnn1, the present study was performed to investigate whether BjAnn1 could function in human GBM cell lines across evolutionary boundaries. With the implication of BjAnn1 in oxidative stress, we aimed at identifying its effect on proliferation and migration in GBM cell lines. Our results demonstrated that, BjAnn1 exhibited a novel inhibitory effect on cell proliferation and migration suggesting it as a potential therapeutic target in the future treatment of glioblastoma.

\section{Materials and Methods \\ Cell culture and reagents}

Human glioblastoma cell lines U251 and U87 were obtained from American Type Culture Collection (Manassas, VA) and cultured in DMEM supplemented with 10\% FBS (Life Technologies, Carlsbad, CA), 50 units $/ \mathrm{mL}$ penicillin and $50 \mu \mathrm{g} / \mathrm{mL}$ streptomycin. Specific antibodies against p50, p65, cyclinD1, c-Myc, SOD2, SOD3, Lamin B, GAPDH, $\beta$-actin and HRP-conjugated secondary antibodies were purchased from Santa Cruz Biotechnology (Santa Cruz, CA). Alexa Fluor conjugated secondary antibodies were obtained from Life Technologies (Carlsbad, CA). Specific epitope-based antibody of BjAnn1 was custom synthesized (IMGENEX, India).

\section{Plasmid construction and stable transfection}

The full-length coding region of Brassica juncea Annexin 1 (BjAnn1) was amplified using sequence-specific primers, BjAnn1 sense 5'-GCGAATTCCACCATGATGGCGACTCT-3', antisense 5'-TCGGATCCTTAAGCATCATCTTCACCG-3' and cloned in mammalian expression vector pcDNA3.0 at EcoRI and BamH1 sites. Transfection experiments were performed with empty pcDNA vector (EV) or pcDNA-BjAnn1 in 6-hour serum-starved U251 and U87 cells using lipofectamine ${ }^{\mathrm{TM}} 2000$ following manufacturer's instructions (Life Technologies). Stable expression clones were selected with G418 as reported earlier [37].

\section{Cell viability and clonogenic assay}

Cell viability was determined by MTT assay in empty vector, $\mathrm{pEV}$ and BjAnn1-expressing cells as described earlier [38]. Briefly, U251 and U87 cells were seeded in 96-well plates $\left(2 \times 10^{3}\right.$ cells/well $)$ in DMEM medium containing $10 \%$ serum. After incubation for $72 \mathrm{~h}$, growth inhibition was determined by using traditional MTT assays. For the clonogenic assays, EV- and BjAnn1-expressing U87 and U251 cells (500 cells/well) were seeded in 6-well plates and allowed to grow for a further period of 8 days. The cells were then fixed in ice cold methanol and stained with $0.5 \%$ crystal violet solution to visualize the colonies.

\section{qRT-PCR and CDNA-PCR array}

Total RNA was isolated from empty vector (pEV) and pcDNABjAnn1 expressingU251 and U87 celllinesusingTRIzol reagentandsemiquantitative RT-PCR analysis were performed as described earlier [39]. The following primers were used for semi-quantitative and quantitative PCR analyses: BjAnn1 sense 5'-ATCTTGCTCTGGACTCTTGAA3 'and antisense 5'-TGGTGTGGTGAGCGACAT-3'and GAPDH sense $5^{\prime}$-GGAGTCAACGGATTTGGTCGTAT-3' and antisense, 5'-GTCTTCACCACCATGGAGAAGGCT-3', respectively. The potential changes in the ROS-related gene expression profile by BjAnn1 overexpression were analyzed by performing the cDNA PCR arrays in EV-and BjAnn1-expressing U87 cells using Human Oxidative Stress and Antioxidant Defense (PAHS065) RT ${ }^{2}$ Profiler PCR Array kits following the manufacturer's instructions (SA Biosciences, Frederick, MD, USA) as described earlier [40]. The following PCR conditions were used: 1 cycle of $95^{\circ} \mathrm{C}$ for $10 \mathrm{~min}$ and 40 cycles of $95^{\circ} \mathrm{C}$ for $15 \mathrm{~s}$, $60^{\circ} \mathrm{C}$ for $30 \mathrm{~s}, 72^{\circ} \mathrm{C}$ for $30 \mathrm{~s}$, followed by 1 cycle of $72^{\circ} \mathrm{C}$ for $10 \mathrm{~min}$. The data obtained from three independent arrays were analyzed using iCycler IQ version 3.1 software (Bio-Rad Laboratories, Hercules, CA, USA) and Ct values were converted into fold change of expression with $2-\Delta \Delta \mathrm{Ct}$ method $(\Delta \Delta \mathrm{CT}=\Delta \mathrm{CT}$ treatment [BjAnn1] $-\Delta \mathrm{CT}$ control $[\mathrm{EV}])$. The gene expression changes in BjAnn1 cells in comparison to EV-controls were determined by fold change values using web-based analysis software and presented in the form of a heat map (http:// pcrdataanalysis.sabiosciences.com/pcr/arrayanalysis.php).

\section{Western blotting}

Whole cell lysates were prepared from GBM cells in modified RIPA buffer ( $150 \mathrm{mM} \mathrm{NaCl}, 50 \mathrm{mM}$ Tris- $\mathrm{HCl}, 50 \mathrm{mM} \mathrm{NaF}, 5 \mathrm{mM}$ EDTA, $0.5 \%$ [wt/vol] sodium deoxycholate and $1 \%$ Triton X-100) containing phosphatase and protease inhibitors [37]. The cytosolic and nuclear extracts were prepared as described earlier [38]. Lysates were run on SDS-PAGE followed by Western blotting and immunoreactivity was visualized using enhanced chemiluminescence detection kit.

\section{Wound-healing migration and Transwell-Matrigel invasion assays}

Wound-healing cell migration and Transwell-matrigel invasion assays were performed as described earlier [40]. Briefly, the EVand BjAnn1-expressing U251 and U87 cells were cultured in 6-well plates $\left(2 \times 10^{5}\right.$ cells/well) overnight and a straight scratch was made in individual wells using $200-\mu$ pipette tip, which has been considered as point $0 \mathrm{~h}$. After 12 hours, plates were observed for wound closure and average cell migration distances were measured using a microscope calibrated with an ocular micrometer. For matrigel invasion assay, Transwell inserts (ThinCertTM; Greiner Bio-One, Monroe, NC) with $8 \mathrm{~mm}$ pores were coated with $200 \mu \mathrm{L}$ growth factor reduced Matrigel $(50 \mu \mathrm{g} / \mathrm{mL}$ in serum-free medium) (Collaborative Research, Inc., Boston, MA) for $2-3 \mathrm{~h}$ by placing in $37^{\circ} \mathrm{C}$ incubator. About $500 \mu \mathrm{L}$ of cell suspension $\left(1 \times 10^{4}\right.$ cells/well) was added in each well. Invaded cells through matrigel after $24 \mathrm{~h}$ were stained with Hema-3 and visualized under light microscope. Percentage of cell invasion and relative migration distance values were represented as mean \pm SE obtained from three experimental replicates. 
Citation: Gangadhara Reddy S, Divya K, Kirti PB, Prakash Babu P (2013) Novel Antiproliferative and Antioxidant Role of BjAnn1, a Mustard Annexin Protein in Human Glioblastoma Cell Lines. J Cancer Sci Ther 5: 256-263. doi:10.4172/1948-5956.1000214

\section{ROS measurement and Immunocytochemistry}

Oxidative stress and superoxide content was determined using total ROS/Superoxide Detection Kit following manufacturers' instructions. In brief, the EV- and BjAnn1-expressing U251 and U87 cells were seeded in 96 -well plates $\left(1 \times 10^{4} /\right.$ well $)$ and supplemented with $10 \mu \mathrm{M}$ $\mathrm{H}_{2} \mathrm{O}_{2}$ for 2 hours in independent experiments as described above in 6-8 replicates. $\mathrm{H}_{2} \mathrm{O}_{2}$-containing medium was replaced with fresh medium and cells were further incubated for another 12-16 hours. Then cells were washed gently with $1 \times$ wash buffer followed by incubation in 100 $\mu \mathrm{l} /$ well of ROS/Superoxide detection mix for $1 \mathrm{~h}$ at $37^{\circ} \mathrm{C}$. Total ROS content was determined by reading culture plates with a fluorescence microplate reader at $488 \mathrm{~nm}-530 \mathrm{~nm}$. Green fluorescence of $\mathrm{H}_{2}$ DCFDA depicting ROS levels was also visualized under a confocal microscope. Immmunocytochemical analysis was performed as described earlier [38]. Briefly, pEV- and BjAnn1-expressing cells were seeded in 4-well chamber slides at a density of $1 \times 10^{4} /$ well and incubated overnight. Cells were fixed with $4 \%$ paraformaldehyde and permeabilized in $0.1 \%$ Triton-X100. Non-specific binding was blocked by treating the cells with $3 \%$ BSA and the cells were incubated with anti-BjAnn1 antibody (1:100 dilutions) overnight at $4^{\circ} \mathrm{C}$. Cells were washed and incubated with Alexa Fluor-conjugated secondary antibodies for 1 hour at room temperature, washed, mounted and observed under a confocal microscope. The nuclei were counterstained with DAPI (4'-6-diamidino-2-phenylindole).

\section{Reporter gene assays}

Reporter gene assays were performed as described earlier [41]. Control (EV) or BjAnn1 expressing U251 and U87 cells were seeded in 6-well plates. After overnight incubation, the cells were transiently transfected with NF- $\kappa$ B-Luc plasmid using lipofectamine ${ }^{\mathrm{TM}} 2000$ for 6 h. After $48 \mathrm{~h}$ of transfection, cells were further treated with TNF- $\alpha$ (10 $\mathrm{ng} / \mathrm{ml}$ ) and incubated for an additional period of $12 \mathrm{~h}$. Each transfection was carried out in triplicate and normalized with the $\beta$-gal activity and total protein concentration. Luciferase activity was measured by using the luciferase assay system (Promega, Madison, WI). Luciferase activity was expressed as percent of relative light units versus untreated transfected cells.

\section{Bioinformatic analyses}

The potential mammalian homologs of BjAnn1 were primarily identified by performing BLAST with amino acid sequence and multiple sequence alignment was performed by with ClustalW2 (http://www.ebi.ac.uk/Tools/msa/clustalw2/) \& Phylogeny.fr [42], http://www.phylogeny.fr/version2_cgi/index.cgi. Conserved annexin domains in different annexin proteins were identified by Sequence SMART [43], http://smart.embl-heidelberg.de/. Aligned individual domains were displayed as sequence-logo plots using WebLogo 3.3 [44], http://weblogo.threeplusone.com/create.cgi.

\section{Statistical analysis}

Data obtained from different sets of treatment groups were statistically analyzed by one-way ANOVA with Tukey's test. The mean \pm SE values from at least three individual repetitions were presented and the significant difference was denoted at $p \leq 0.01$.

\section{Results}

\section{Brassica juncea Annexin 1 (BjAnn1) is a close homolog to animal annexins}

Annexin proteins are widely conserved in plants and animals.
Sequence analyses revealed that the mustard annexin BjAnn1 shows the most striking amino acid sequence homology up to $73.18 \%$ with human annexin A13 (Figure 1A). BjAnn1 also showed significant sequence homology with human annexins A2 (70.66\%), A3 (72.53\%) and A8 (70.03\%). All annexins are characterized by the presence of a core domain made up of four similar repeats of approximately 70 amino acids long. The four annexin repeats are packed to form a flattened disc-like structure with a slight convex surface on which the type II and type III $\mathrm{Ca}^{2+}$ binding loops are located and a concave surface where the $-\mathrm{NH}_{2}$ and $-\mathrm{COOH}$ termini come side-by-side $[17,45]$. To analyze the potential homology between the four conserved annexin repeats, we performed a domain-specific sequence alignment and generated sequence logos using WebLogo 3.3 program (Figure 1B). Several conserved and semi-conserved residues were identified in all four annexin motifs.

\section{Ectopic expression of BjAnn1 in human GBM cell lines}

Our earlier studies demonstrated the peroxidase activity of mustard annexin (BjAnn1) and its beneficial effects in alleviating oxidative

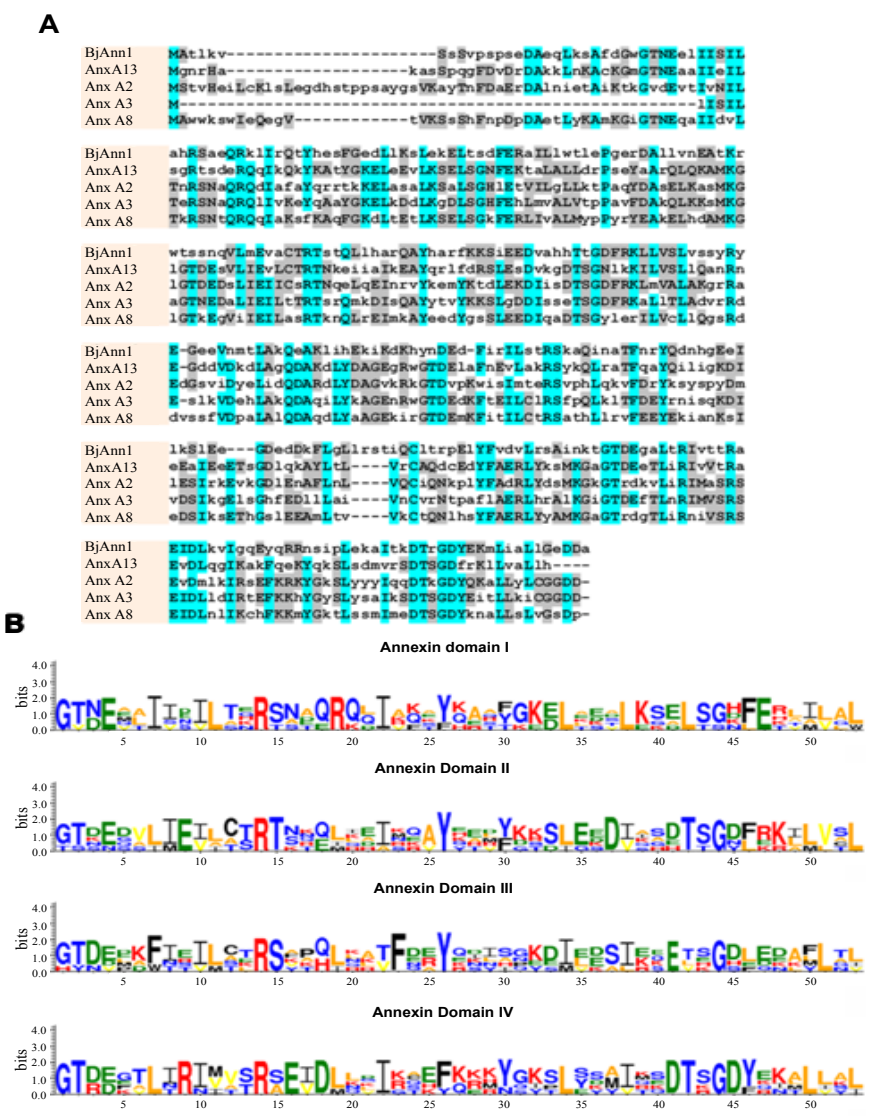

Figure 1: Sequence alignment of Brassica juncea annexin 1 (BjAnn1) with human annexins. (A) The amino acid sequences of BjAnn1 (ABB59547) and human annexins A2 (AAH23990), A3 (CAG28576.1), A8 (AAH73755.1) and A13 (CAA77578.1) were aligned using ClustalW2 and Phylogeny.fr. The blue and gray highlighting indicates conserved and semi-conserved substitutions, respectively, in the multiple sequence alignment. (B) Representative four annexin domains (domain I: 28-80aa, domain II: 100-152aa, domain III: 183-232aa and domain IV: 259-311aa) in BjAnn1 and human annexins A2, A3, A8 and A13 were identified by SMART sequence analyses. Sequence logos were created by subjecting the domain-specific sequence alignment as input to WebLogo 3.3 program (http://weblogo.threeplusone.com/create.cgi). The height of each amino acid directly reflects the degree of conservation and frequency of that amino acid in the alignment. 
stress upon overexpression in transgenic tobacco and cotton plants from oxidative stress $[24,25]$. GBM cells have been shown to exhibit high ROS levels when compared to their normal counterparts and ROS is essential in maintaining increased cell proliferation, migration and invasion in tumors. In the present investigation, we aimed at verifying the effect of ROS-scavenging BjAnn1 expression in GBM cells. To accomplish this, we ectopically expressed BjAnn1 in human U251 and U87 GBM cells. The stable expression of BjAnn1 was confirmed by Western blotting (Figure 2A) and RT-PCR (Figure 2B). To identify the sub-cellular localization of BjAnn1, immunofluorescence analyses was performed. The BjAnn1 protein was primarily localized in the membrane and cytoplasmic regions in the GBM cell lines. These results are in corroboration with the cellular localization of human annexins, which possess phospholipid and F-actin-binding activity and display abundant membrane localization (Figure 2C).

\section{BjAnn1 expression inhibited proliferation, colony formation, migration and invasion in U251 and U87 GBM cells}

To study the role of BjAnn1 on proliferation, an MTT assay was performed. In comparison to EV-treated controls, BjAnn1expressing U251 and U87 cells showed significance decrease in cell proliferation (Figure 3A). Further, clonogenic assay revealed that colony-forming ability of $\mathrm{BjAnn1-expressing} \mathrm{cells} \mathrm{was} \mathrm{prominently}$ inhibited in comparison to EV-controls (Figure 3B). It is well evident that glioma cells possess high migratory and invasive potential and often metastasized into surrounding normal brain parenchyma. We next determined the effect of BjAnn1 expression on migration and invasion of U87 and U251 cells by performing scratch wound-healing assay and matrigel invasion assay respectively. As shown in Figure 3C, the migratory potential of BjAnn1-expressing cells was significantly decreased when compared to $\mathrm{pEV}$-expressing cells. Correspondingly, the invasive potential through matrigel was significantly inhibited in U87 and U251 upon BjAnn1 expression (Figure 3D). These results suggest the anti-proliferative and anti-migratory effect of BjAnn1 in GBM cells.

\section{$B j A n n 1$ reduced ROS levels and modulated the expression of oxidative stress and antioxidant defense genes}

Characteristic traits of deregulated cell proliferation and
A

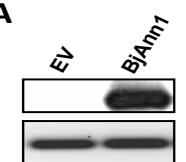

U251

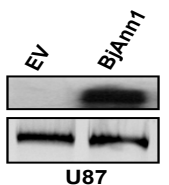

BjAnn1 GAPDH

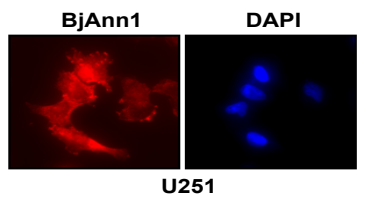

B

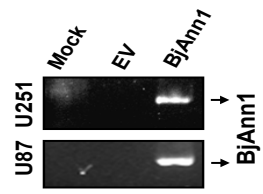

BjAnn1

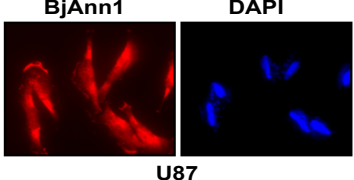

Figure 2: Confirmation of stable BjAnn1 expression in human GBM cell lines. (A) Whole cell lysates from EV- and BjAnn1-expressing cells were subjected to Western blotting. Blots were re-probed with GAPDH to monitor equal loading. (B) Semi-quantitative RT-PCR was performed in EV- and BjAnn1-expressing U251 and U87 cells. (C) Confocal microscopy was performed as described in Materials and Methods. Representative micrographs of BjAnn1 (red) expression were presented from randomly selected microscopic fields. Nuclei were counterstained with DAPI.

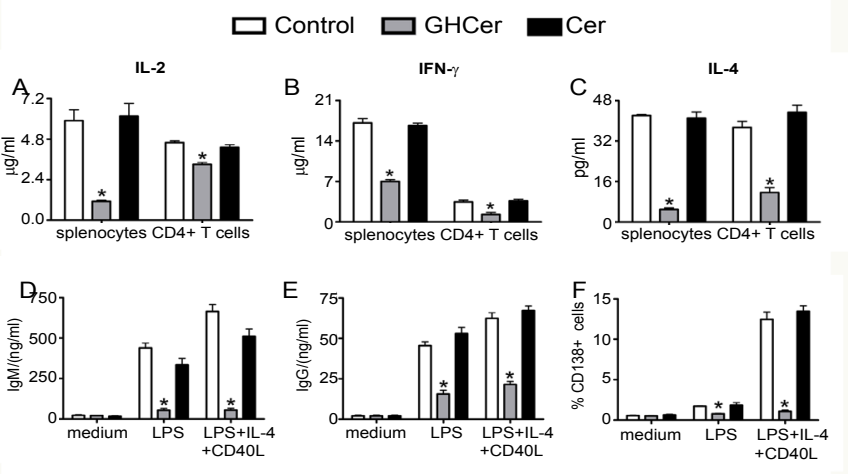

Figure 3: Ectopic expression of BjAnn1 reduces the proliferation, migration and colony formation of GBM cell lines. (A) U251 and U87 cells were transfected with control ( $p E V$ ) and BjAnn1 plasmids and the proliferation was determined at 72 $h$ by using the MTT assay. All data presented are the mean of 3 independent experiments with mean \pm SE. ${ }^{*}, p<0.05$. (B) U251 and U87 cells stably expressing control (pEV) or BjAnn1 plasmids were seeded in 6 well plates (500 cells/well) and allowed to grow for 8 days. Colonies were stained with crystal violet and colonies that contain $\geq 50$ cells were counted. All data presented are the mean of 3 independent experiments with mean \pm SE. *,$p<0.05$. (C) U251 and U87 GBM cells were transfected with control $(\mathrm{pEV})$ and BjAnn1 plasmids were subjected scratch wound healing assay as described in materials and methods and the distance of migration was determined after 12 hours. (D) U251 and U87 cells transfected with control $(\mathrm{pEV})$ and BjAnn1 plasmids were subjected to matrigel invasion assay and the percentage of invaded cells compared to control were plotted on histogram.

invasiveness were in part attributed to the high ROS levels and ROSdependent signaling activation in tumors. Based on the previous reports showing peroxidase activity of BjAnn1, we next determined its effect on ROS levels in U87 and U251 cells. The BjAnn1-expressing cells showed a significant reduction in ROS levels compared to the mock- or pEV-controls (Figure 4A). Based on this interesting result of significant ROS diminution, we attempted to analyze the possible modulation in expression levels of genes responsive to ROSdependent signaling and metabolism. To achieve this, we performed a human oxidative stress and antioxidant defense PCR array. BjAnn1 expressing cells showed prominent transcriptional upregulation of Superoxide dismutase copper chaperone (CCS), Cytochrome b (CYB), NADPH oxidase (DUOX2), Forkhead box M1 (FOXM), Glutathione synthetase (GSS), Mannose-binding lectin (protein C) (MBL2), Metallothionein 3 (MT3), Metallothionein-like 5 (MTL5), Peroxidasin homolog (PXDN), Mn superoxide dismutase (SOD2) and $\mathrm{Cu} / \mathrm{Zn}$ extracellular superoxide dismutase (SOD3) genes, which are involved in superoxide metabolism, apoptosis, transcriptional regulation and signal transduction (Figure 4B). The expression of genes upregulated in response to BjAnn1 expression was summarized in Table 1. With the striking transcriptional elevation of SOD2 (MnSOD) and SOD3 $(\mathrm{Cu} / \mathrm{ZnSOD})$ in BjAnn1 expressing GBM cells, we further checked the protein levels. Ectopic expression of BjAnn1 significantly elevated SOD2 and SOD3 expression levels in both cell lines (Figure 4C). To confirm the specificity of BjAnn1 function in alleviating ROS in GBM cells and to rule out the off-target consequences, we treated U87 cells with $\mathrm{H}_{2} \mathrm{O}_{2}$. Notably, treatment with $\mathrm{H}_{2} \mathrm{O}_{2}$ significantly enhanced $\mathrm{ROS}$ levels in control and EV-treated cells (Figure 4D). On the other hand, 
Citation: Gangadhara Reddy S, Divya K, Kirti PB, Prakash Babu P (2013) Novel Antiproliferative and Antioxidant Role of BjAnn1, a Mustard Annexin Protein in Human Glioblastoma Cell Lines. J Cancer Sci Ther 5: 256-263. doi:10.4172/1948-5956.1000214

A

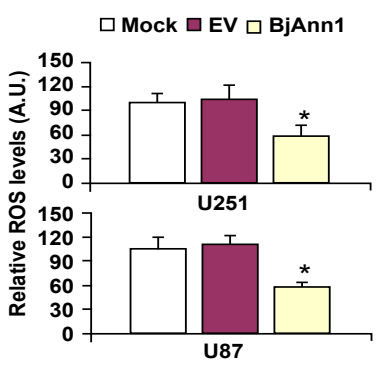

C

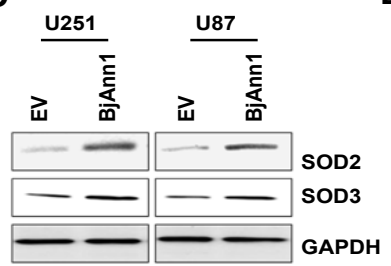

B
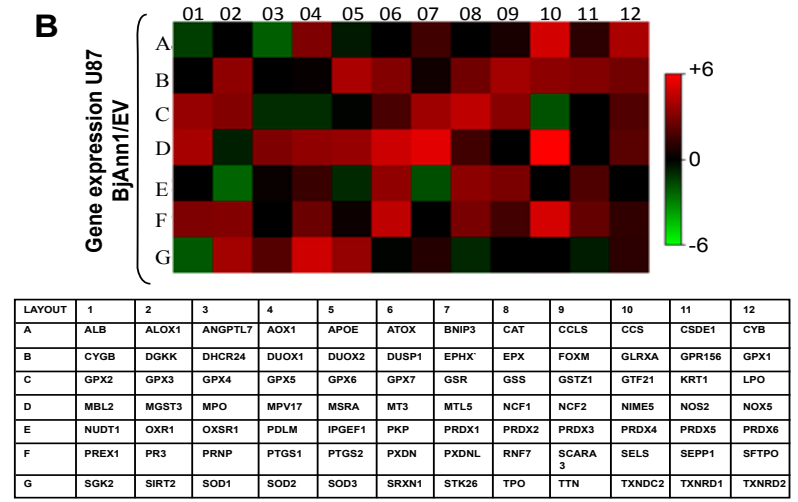

D

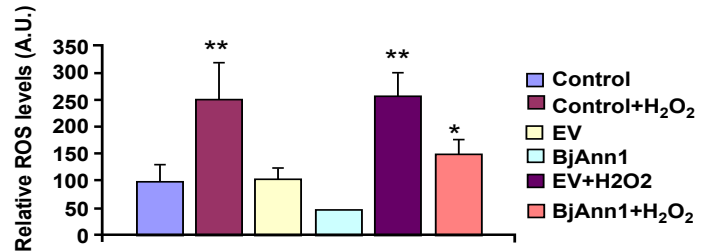

Figure 4: BjAnn1 exhibits ROS scavenging activity and modulates the expression of genes involved in oxidative stress and antioxidant defense. (A) U251 and U87 cells were transfected with EV- and BjAnn 1 plasmids and after $48 \mathrm{~h}$ cells were seeded in 96 -well plates $\left(1 \times 10^{4} /\right.$ well) and ROS levels were determined using total ROS/ Superoxide Detection Kit following manufacturers' instructions. (B) Potential changes in the multiple gene expression profile in BjAnn1-expressing U87 cells were determined in comparison to EV-treated cells using Human Oxidative Stress and RT ${ }^{2}$ Profiler Human Antioxidant Defense arrays as explained in Materials and Methods. The relative fold changes values of gene expression in BjAnn1-expressing cells in comparison to EV-treated controls were obtained from three experimental replicates and represented in the form of a heat map. (C) Whole cell lysates were prepared from control (EV) and BjAnn1 expressing U251 and U87 cells and the expression levels of SOD2 and SOD3 were determined by western blotting. (D) U87 cells transfected with EV- and BjAnn1 plasmids were seeded in 96 -well plates (1 $10^{4} /$ well) and treated with $10 \mu \mathrm{M} \mathrm{H}_{2} \mathrm{O}_{2}$ for 2 hours, then fresh medium was added and further incubated for another 12-16 hours. Cells were incubated in $100 \mu \mathrm{l} /$ well of ROS/Superoxide detection mix for $1 \mathrm{~h}$ at $37^{\circ} \mathrm{C}$. Total ROS content was determined by reading culture plates with a fluorescence microplate reader at $488 \mathrm{~nm}-530 \mathrm{~nm}$.

\begin{tabular}{|c|c|c|c|}
\hline Gene & NCBI REFSEQ & Description & Fold change: BjAnn1/EV \\
\hline AOX1 & NP_001150.3 & Aldehyde oxidase 1 & 3.13 \\
\hline CCS & NP_005116.1 & Superoxide dismutase copper chaperone & 5.03 \\
\hline CYB & NP_536855.1 & Cytochrome b & 4.25 \\
\hline DGKK & NP_001013764.1 & Diacylglycerol kinase, kappa & 3.51 \\
\hline DUOX2 & NP_054799.4 & NADPH oxidase & 4.28 \\
\hline DUSP1 & NP_004408.1 & Dual specificity phosphatase 1 & 3.23 \\
\hline FOXM & NP_001230017.1 & Forkhead box M1 & 4.04 \\
\hline GPR156 & NP_001161743.1 & G protein-coupled receptor 15 & 3.43 \\
\hline GPX1 & NP_000572.2 & Glutathione peroxidase 1 & 3.22 \\
\hline GPX2 & NP_002074.2 & Glutathione peroxidase 2 & 3.97 \\
\hline GPX3 & NP_002075.2 & Glutathione peroxidase 3 & 3.26 \\
\hline GSR & NP_000628.2 & Glutathione reductase & 3.96 \\
\hline GSS & NP_000169.1 & Glutathione synthetase & 4.95 \\
\hline GSTZ1 & NP_001504.2 & Glutathione S-transferase zeta 1 & 3.05 \\
\hline MBL2 & NP_000233.1 & Mannose-binding lectin (protein C) 2, soluble & 4.20 \\
\hline MPO & NP_000241.1 & Myeloperoxidase & 3.16 \\
\hline MPV17 & NP_002428.1 & MpV17 mitochondrial inner membrane protein & 3.55 \\
\hline MSRA & NP_001129142.1 & Methionine sulfoxide reductase $A$ & 3.75 \\
\hline MT3 & NP_005945.1 & Metallothionein 3 & 5.43 \\
\hline MTL5 & NP_004914.2 & Metallothionein-like 5, testis-specific (tesmin) & 6.34 \\
\hline NME5 & NP_003542.1 & NME/NM23 family member & 7.74 \\
\hline PKP & NP_000290.2 & Plakophilin 1 & 3.59 \\
\hline PRDX2 & NP_005800.3 & Peroxiredoxin 2 & 3.44 \\
\hline PRDX3 & NP_006784.1 & Peroxiredoxin 3 & 3.03 \\
\hline PREX1 & NP_065871.2 & Ptdlns(3,4,5)-dependent Rac exchanger 1 & 3.14 \\
\hline PR3 & NP_002768.3 & Proteinase 3 & 3.23 \\
\hline PXDN & NP_036425.1 & Peroxidasin homolog & 5.33 \\
\hline SELS & NP_060915.2 & Selenoprotein S & 7.04 \\
\hline SIRT2 & NP_001180215.1 & Sirtuin 2 & 3.72 \\
\hline SOD2 & NP_000627.2 & Mn superoxide dismutase & 6.42 \\
\hline SOD3 & NP_003093.2 & Extracellular superoxide dismutase [Cu-Zn] & 3.94 \\
\hline
\end{tabular}

Table 1: Modulation of oxidative stress response genes in BjAnn1-expressing cells. Total RNA was isolated from EV-and BjAnn1-stable expressing cells and cDNAs were subjected to Human Oxidative and Antioxidant Defense PCR array (PAHS65) (SABiosciences, Valencia, CA) using real-time PCR. The fold change values of gene expression were determined in BjANN1 cells in comparison to EV-treated controls in three individual experiments using web-based software (http://www.sabiosciences. com/pcrarraydataanalysis.php). 
BjAnn1 expression significantly reversed and decreased the $\mathrm{H}_{2} \mathrm{O}_{2}$ induced ROS levels. These results clearly show the ROS-scavenging activity of BjAnn1 and suggest its role in alleviating oxidative stress in GBM cells.

\section{BjAnn1 attenuated the NF-kB nuclear translocation and its activation}

Deregulated NF- $\kappa B$ signaling is a hallmark in GBM progression. $N F-\kappa B$ is known to be activated in response to ROS and critical in the maintenance of tumor cell proliferation and migration. With the compromised ROS levels upon BjAnn1 expression in GBM cells, we next proceeded to check the levels of NF- $\mathrm{kB}$ subunits, p65 and p50. As shown in Figure 5A, the TNF- $\alpha$ induced p50 and p65 nuclear translocation was significantly reduced in BjAnn1 expressing cells compared to pEV-control cells. In contrast, the cytoplasmic p65 levels were high in BjAnn1 expressing cells compared to pEV-control cells. To further study whether BjAnn1 interfered with the NF- $\kappa B$ pathway activation, we performed gene reporter assays. As shown in Figure $5 B$, the BjAnn1 overexpression significantly reduced the NF- $\kappa B$ reporter activity compared to pEV-control cells. Further, we also checked the expression of proliferation markers such as cyclin D1 and c-Myc, the direct transcriptional target genes of NF- $\mathrm{kB}$. The expression levels of cyclin D1 and c-Myc were down regulated in BjAnn1 expressing GBM cells compared to control GBM cells (Figure 5C). These results clearly demonstrated that the expression of BjAnn1 inhibits the GBM cell proliferation by interfering with NF- $\kappa \mathrm{B}$ pathway activation.

\section{Discussion}

Despite using the advanced multimodal treatments, GBM remain to be the deadliest among the tumors of the central nervous system. Since the prognosis remains poor in GBM, there is an imperative need to develop novel and alternative therapeutic regimens in their control. Recently, increasing evidences suggest the possible functions of plant genes across evolutionary boundaries in the potential therapeutic applications in mammalian systems in vitro and in vivo [37,46]. Earlier studies demonstrated the novel roles of plant proteins in the regulation of cell proliferation when heterologously expressed in a variety of cancer cells $[22,23,46]$. Recently, we showed that expression of mammalian IкB homologue, NPR1 (non-expressor of pathogenesis related protein 1) from mustard in GBM cells inhibited their proliferation via the inhibition of NF- $\kappa \mathrm{B}$ pathway [37]. The Thymidine kinase 1 from Tomato exhibited tumor suppressive role when expressed in human GBM cells and enhanced sensitivity to cytotoxic agents [46]. In line with these reports and based on our earlier experiments showing the in vitro peroxidase activity of BjAnn1, we attempted to verify the effect of BjAnn1 expression in human GBM cell lines. We showed that overexpression of $\mathrm{BjAnn} 1$ reduced proliferation, migration and invasion of GBM cells and reduced the ROS generation with the enhanced expression of antioxidant superoxide dismutases, SOD2 and SOD3. Further, BjAnn1 overexpression inhibited the activation of NF$\kappa B$ pathway in GBM cells.

The Brassica Juncea Annexin 1 (BjAnn1) expression in tobacco and cotton conferred oxidative stress tolerance and increased disease
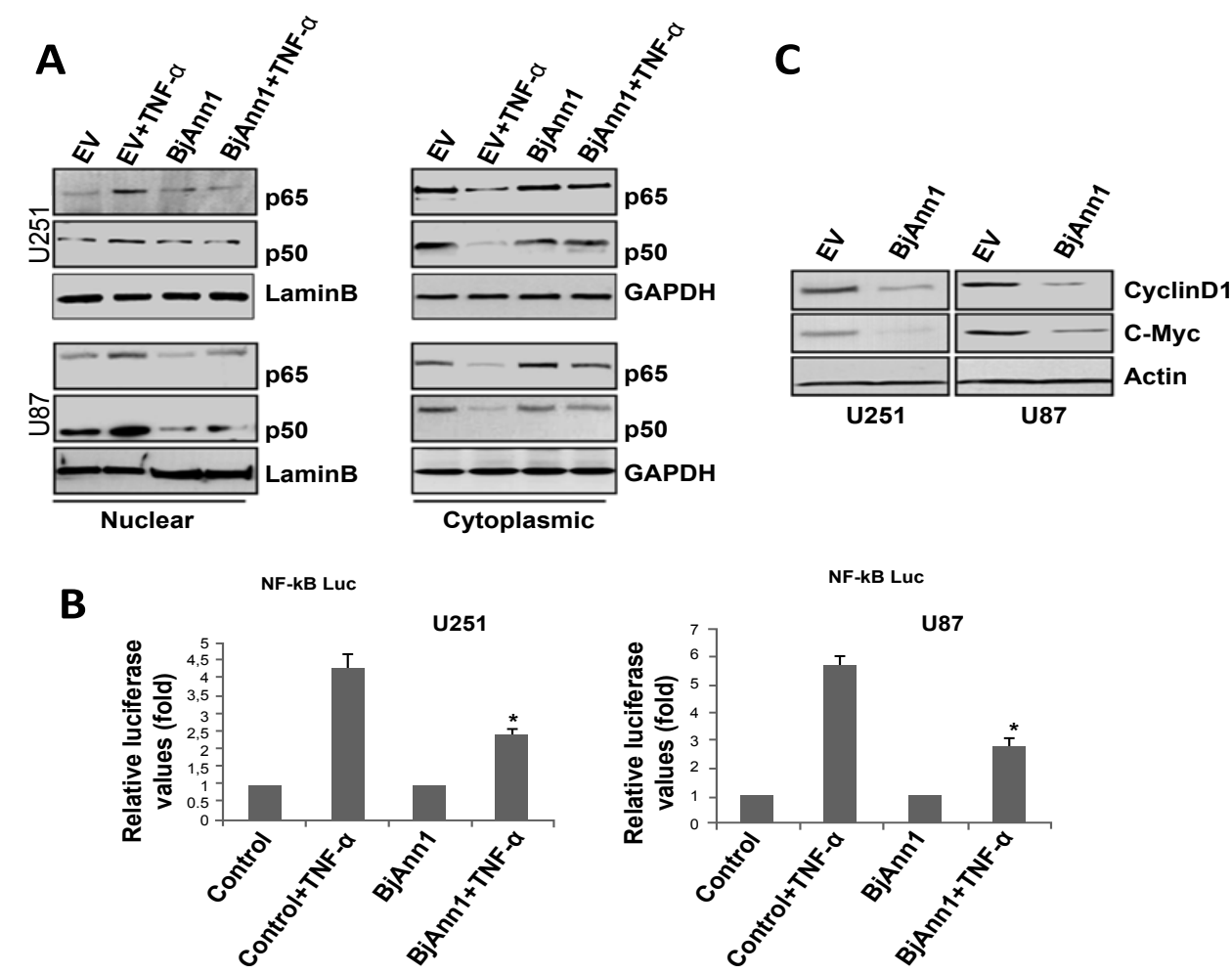

Figure 5: BjAnn1 attenuated the nuclear translocation of NF-kB and reduces its target gene expression. (A) Cytoplasmic and Nuclear lysates were prepared from U251 and U87 cells that are stably expressing control (EV) and BjAnn1 plasmids and stimulated with or without TNF- $\alpha$ for 30 min and subjected to Western blot analysis with p65 and p50 antibodies. GAPDH and Lamin B were used as loading controls for cytoplasmic and nuclear fractions respectively. (B) U251 and U87 cells stably expressing control $(\mathrm{pEV})$ and BjAnn1 were transfected with the NF-kB-Luc reporter and after $48 \mathrm{~h}$ cells were stimulated with TNF- $\alpha$ and the luciferase activity was measured. All data presented are the mean of 3 independent experiments with mean \pm SE. *,$p<0.05$, t test. (C) Whole cell lysates were prepared from U251 and U87 cells that are stably expressing control (EV) and BjAnn1 plasmids subjected to Western blot analysis with cyclin D1 and c-Myc antibodies. $\beta$-actin used as an loading control. 
resistance [23,24]. In both the transgenic plants, ROS scavenging activities were demonstrated. The Oxy5/AtAnn1 protein from Arabidopsis thaliana displayed peroxidase activity and protected oxidative stress-susceptible OxyR-mutant of E.coli from $\mathrm{H}_{2} \mathrm{O}_{2}$ induced death [21]. In another study, overexpressed $\mathrm{Oxy} 5$ in $\mathrm{CHO}$ cells and mouse leukemic K36 cells decreased superoxide production, PKC activity and inhibited subcutaneous tumor growth in nude mice [23]. Upon expression in TNF-sensitive HeLa D98 cells, Oxy5 conferred resistance to TNF cytotoxicity and decreased TNF-induced intracellular superoxide formation [22]. It has also been shown that annexins exhibited ROS neutralizing activity [20]. These studies supported the antioxidant role of annexin proteins in heterologous systems. In our present study, we ectopically expressed the BjAnn1 in GBM cells and found that it is localized mostly to the cell membrane and cytoplasm. It has been shown that annexins in eukaryotes bind to F-actin elements, which keep the annexins at cell membrane. The observed membrane staining of BjAnn 1 could possibly be due to its binding to F-actin elements.

Plant annexins are expressed throughout the life cycle and their expression is influenced by the environmental cues. It is evident that annexins are capable of linking $\mathrm{Ca}^{+}$, redox and lipid signaling to coordinate the development with responses to the biotic and abiotic environment $[19,47]$. In the present study, phylogenetic analysis of BjAnn 1 showed $\sim 73 \%$ homology to human annexin A13. Annexin A13 is considered the original founder gene of the vertebrate 12 member annexin A family [44,45] and it is believed to be an earliest branching subfamily in vertebrates and physically non-syntenic to other human annexins $[48,49]$.

In mammalian systems, annexins perform several functions including key roles in carcinogenesis. The expression profiles of annexins are consistently different in various neoplasms with overexpression in specific types of cancers, while others show consistent loss of expression. Several other studies showed that the changes in annexin expression and their subcellular localization contribute to the development and progression of cancer [13]. In our study, we demonstrated that overexpression of BjAnnl reduced the proliferation, migration and invasion of GBM cells. Further, BjAnn1 overexpression reduced the ROS generation in GBM cells suggesting its evolutionarily conserved ROS scavenging activity. It has been shown that glioma cells express antioxidant enzymes such as superoxide dismutases, catalase and glutathione peroxidase [4]. Oxidative stress is one of the key steps in the progression of carcinogenesis. Antitumor role of antioxidant enzymes was evident in gliomas. In addition, cancer cells have low levels of antioxidant enzymes when compared to their normal cell counter parts $[4,33]$. The tumor suppressive role of MnSOD (SOD2) and $\mathrm{Cu} / \mathrm{ZnSOD}$ (SOD3) was earlier reported in several tumors including glioma $[5,7,35,36]$. Overexpression of MnSOD reduces the malignant phenotype and glioma tumor growth, and generally considered as tumor suppressor in gliomas [35]. In another study when $\mathrm{Cu} / \mathrm{ZnSOD}$ was overexpressed, tumor growth and metastasis were reduced in mice [5]. Inhibition of $\mathrm{Cu} / \mathrm{ZnSOD}$ leads to the increased motility of cancer cells. MnSOD catalyzes the dismutation of superoxide radical to hydrogen peroxide and molecular oxygen. In our study, we investigated the changes in the expression of genes related to oxidative stress, which includes peroxidases, superoxide dismutases, oxidative stress responsive genes, antioxidants and genes involved in ROS metabolism. We found a significant deviation of transcriptional levels of oxidative stress responsive genes in BjAnn1 expressing GBM cells. We also demonstrated the increased expression of tumor suppressive antioxidant enzymes, MnSOD and $\mathrm{Cu} / \mathrm{ZnSOD}$ in BjAnn1 expressing GBM cells. It has also been shown that ROS activates the NF- $\kappa B$ [26] and NF- $\kappa B$ pathway constitutively activated in gliomas. We hypothesize that the reduced proliferative and migration properties of BjAnn1 exert partly through inhibition of NF- $\mathrm{B}$ p pathway. In our study we observed that the nuclear translocation of p50 and p65 was also reduced in BjAnn1 overexpressing cells. Further, NF-kB reporter gene activity and its target gene activation such as cyclin D1 and c-Myc were reduced in BjAnn1 expressing GBM cells.

In summary, our study for the first time demonstrated that heterologous expression of $\mathrm{BjAnn} 1$ inhibits the proliferation, migration and invasion of GBM cells by enhancing ROS scavenging activity with concomitant activation of antioxidant enzymes and inhibition of NF$\kappa \mathrm{B}$ pathway. This study may initiate the development of new treatment modalities using plant transgenes as therapeutic applications for glioblastoma.

\section{Acknowledgements}

The authors thank DBT, CSIR, DST, Government of India, New Delhi, for financial assistance.

\section{References}

1. Jena NR (2012) DNA damage by reactive species: Mechanisms, mutation and repair. J Biosci 37: 503-17.

2. Gille JJ, van Berkel CG, Joenje H (1994) Mutagenicity of metabolic oxygen radicals in mammalian cell cultures.Carcinogenesis 15: 2695-2699.

3. Mates JM, Segura JA, Alonso FJ, Marquez J(2012) Oxidative stress in apoptosis and cancer: an update.Arch Toxicol 86: 1649-65.

4. Zhong W, Yan T, Lim R, Oberley LW (1999) Expression of superoxide dismutases, catalase, and glutathione peroxidase in glioma cells. Free Radic Biol Med 27: 1334-1345.

5. Zhang Y, Zhao W, Zhang HJ, Domann FE, Oberley LW (2002) Overexpression of copper zinc superoxide dismutase suppresses human glioma cell growth Cancer Res 62: 1205-1212.

6. Tochhawng L, Deng S, Pervaiz S, Yap CT (2012) Redox regulation of cancer cell migration and invasion. Mitochondrion 12: 246-253.

7. Hu Y, RosenDG, Zhou Y, Feng L, Yang G, et al.(2005) Mitochondria manganese-superoxide dismutase expression in ovarian cancer: role in cell proliferation and response to oxidative stress. J BiolChem 280: 39485-39492.

8. Zhu P, Tan MJ, Huang RL, TanCK, Chong HC, et al. (2011) Angiopoietin-like 4 protein elevates the prosurvival intracellular $\mathrm{O}_{2}(-): \mathrm{H}_{2} \mathrm{O}_{2}$ ratio and confers anoikis resistance to tumors. Cancer Cell 19: 401-415.

9. Shi X, Zhang Y, Zheng J, Pan J (2012) Reactive Oxygen Species in Cancer Stem Cells.Antioxid Redox Signal 16: 1215-1228

10. Morgan RO, Martin-Alemedina S, Iglesias JM, Gonzalez-Florez MI, Fernandez MP (2004) Evolutionary perspective on annexin calcium-binding domains. BiochimBiophysActa 1742: 133-140.

11. Laohavisit A, Davies JM (2009) Multifunctional annexins. Plant Science 177 532-539.

12. Jami, SK, Clark GB, Ayele BT, Ashe P, Kirti PB (2012) Genome wide analysis of Annexinsupergen family in plants. PLoS One 7: e47801.

13. Mussunoor S, Murray GI (2008) The role of annexins in tumour development and progression.J Pathol 216: 131-140.

14. Monastyrskaya K, Babiychuk EB, Draeger A (2009) Theannexins: spatial and temporal coordination of signaling events during cellular stress. Cell Mol Life Sci 66: 2623-2642.

15. Mortimer JC, Laohavisit A, Macpherson N, Webb A, Brownlee C, et al. (2008) Annexins: multifunctional components of growth and adaptation. J Exp Bot 59 : 533-544.

16. Rescher U, Gerke V (2004) Annexins-unique membrane binding proteins with diverse functions. J Cell Sci 117: 2631-2639. 
Citation: Gangadhara Reddy S, Divya K, Kirti PB, Prakash Babu P (2013) Novel Antiproliferative and Antioxidant Role of BjAnn1, a Mustard Annexin Protein in Human Glioblastoma Cell Lines. J Cancer Sci Ther 5: 256-263. doi:10.4172/1948-5956.1000214

17. Gerke V, Moss SE (2002) Annexins: From structure to function. Physiol Rev 82: $331-371$

18. Mortimer JC, Coxon KM, Laohavisit A, Davies JM (2009) Heme-independent soluble and membrane-asosciated peroxidase activity of a Zea mays annexin preparation. Plant Signal Behav. 4: 428-430

19. Laohavisit A, Brown AT, Cicuta P, Davies JM (2010) Annexins: components of the calcium and reactive oxygen signaling network. Plant Physiol. 152: 18241829.

20. Clark G, Konopka-Postupolska D, Hennig J, Roux S (2010) Is annexin 1 a multifunctional protein during stress responses? Plant Signal. Behav 5: 303307.

21. Gidrol X, Sabelli PA, Fern YS, Kush AK (1996) Annexin-like protein from Arabidopsis thaliana rescues delta oxyR mutant of Escherichia coli from $\mathrm{H}_{2} \mathrm{O}_{2}$ stress. ProcNatlAcadSci U S A 93: 11268-11273.

22. Janicke RU, Porter AG, Kush A (1998) A novel Arabidopsis thaliana protein protects tumor cells from tumor necrosis factor-induced apoptosis. BiochimBiophysActa 1402: 70-78.

23. Kush A, Sabapathy K (2001) Oxy5, a novel protein from Arabidopsis thaliana, protects mammalian cells from oxidative stress. Int J Biochem Cell Biol 33: 591602.

24. Divya K, Jami SK, Kirti PB (2010) Constitutive expression of mustard annexin, BjAnn1 enhances abiotic stress tolerance and fiber quality in cotton under stress. Plant MolBiol 73: 293-308.

25. Jami SK, Clark GB, Turlapati SA, Handley C, Roux SJ, et al. (2008) Ectopic expression of an annexin from Brassica juncea confers tolerance to abiotic and biotic stress treatments in transgenic tobacco. Plant PhysiolBiochem 46: 1019-1030.

26. Ohgaki H, Kleihues $P$ (2009) Genetic alterations and signaling pathways in the evolution of gliomas. Cancer Sci 100: 2235-2241.

27. Louis DN, Ohgaki H, Wiestler OD, Cavenee WK, Burger PC, et al. (2007) The 2007 WHO classification of tumours of the central nervous system. ActaNeuropathol 114: 97-109.

28. Dunn GP, Rinne ML, Wykosky J, Genovese G, Quayle SN, et al. (2012) Emerging insights into the molecular and cellular basis of glioblastoma. Genes Dev 26: 756-784.

29. Gloire G, Legrand-Poels S, Piette J (2006) NF-kappaB activation by reactive oxygen species: fifteen years later. BiochemPharmacol 72: 1493-1505.

30. Nagai S, Washiyama K, Kurimoto M, Takaku A, Endo S, et al. (2002) Aberrant nuclear factor-kappaB activity and its participation in the growth of human malignant astrocytoma. J Neurosurg 96: 909-917.

31. Wang CY, Cusack JC Jr, Liu R, Baldwin AS Jr (1999) Control of inducible chemoresistance: enhanced anti-tumor therapy through increased apoptosis by inhibition of NF-kappaB. Nat Med 5: 412-417.

32. Sareddy GR, Geeviman K, Ramulu C, Babu PP (2012) Thenonsteroidal antiinflammatory drug celecoxib suppresses the growth and induces apoptosis of human glioblastoma cells via the NF-KB pathway. J Neurooncol 106: 99-109.

33. Oberley LW, Buettner GR (1979) Role of superoxide dismutase in cancer: a review. Cancer Res 39: 1141-1149.
34. Trachootham D, Alexandre J, Huang P (2009) Targeting cancer cells by ROS mediated mechanisms: a radical therapeutic approach? Nat Rev Drug Discov 8: 579-91.

35. Zhong W, Oberley LW, Oberley TD, St Clair DK (1997) Suppression of the malignant phenotype of human glioma cells by overexpression of manganese superoxide dismutase. Oncogene 14: 481-490.

36. Oberley LW (2005) Mechanism of the tumor suppressive effect of MnSOD overexpression. Biomed Pharmacother 59: 143-148.

37. Kesanakurti D, Sareddy GR, Babu PP, Kirti PB (2009) Mustard NPR1, mammalian IkappaB homologue inhibits NF-kappaB activation in human GBM cell lines. BiochemBiophys Res Commun 390: 427-433.

38. Kesanakurti D, Chetty C, Bhoopathi P, Lakka SS, Gorantla B, Tsung AJ, Rao JS (2011) Suppression of MMP-2 attenuates TNF- $\alpha$ induced NF-KB activation and leads to JNK mediated cell death in glioma. PLoS One. 6:e19341.

39. Sareddy GR, Nair BC, Krishnan SK, Gonugunta VK, Zhang QG, et al (2013) KDM1 is a novel therapeutic target for the treatment of gliomas. Oncotarget 4: 18-28.

40. Kesanakurti D, Chetty C, RajasekharMaddirela D, Gujrati M, Rao JS (2013) Essential role of cooperative NF-kB and Stat3 recruitment to ICAM-1 intronic consensus elements in the regulation of radiation-induced invasion and migration in glioma. Oncogene. doi: 10.1038/onc.2012.546. [Epub ahead of print].

41. Sareddy GR, Nair BC, Gonugunta VK, Zhang QG, Brenner AJ, et al. (2012) Therapeutic significance of estrogen receptor $\beta$ agonists in gliomas. Mol Cancer Ther 11: 1174-82.

42. Dereeper A, Guignon V, Blanc G, Audic S, Buffet S, et al. (2008) Phylogeny. fr: robust phylogenetic analysis for the non-specialist. Nucleic Acids Res 36 : W465-469.

43. Schultz J, Milpetz F, Bork P, Ponting CP (1998) SMART simple modular architecture research tool: identification of signaling domains. ProcNatlAcadSci U S A. 95: 5857-64.

44. Crooks GE, Hon G, Chandonia JM, Brenner SE (2004) WebLogo: A sequence logo generator. Genome Research 14: 1188-1190.

45. Iglesias JM, Morgan RO, Jenkins NA, Copeland NG, Gilbert DJ, et al. (2002) Comparative genetics and evolution of annexin A13 as the founder gene of vertebrate annexins. MolBiolEvol 19: 608-618.

46. Khan Z, Knecht W, Willer M, Rozpedowska E, Kristoffersen P, et al. (2010) Plant thymidine kinase 1: a novel efficient suicide gene for malignant glioma therapy. NeuroOncol 12: 549-558.

47. Laohavisit A, Davies JM (2011) Annexins. New Phytol. 189: 40-53.

48. Braun EL, Kang S, Nelson MA, Natvig DO (1998) Identification of the first fungal annexin: analysis of annexin gene duplications and implications for eukaryotic evolution. J MolEvol 47: 531-543.

49. Morgan RO, Bell DW, Testa JR, Fernandez MP (1998) Genomic locations of ANX11 and ANX13 and the evolutionary genetics of human annexins. Genomics 48: 100-110. 